\title{
Cep63 knockout inhibits the malignant phenotypes of papillary thyroid cancer cell line TPC-1
}

\author{
CHENGUANG LIU ${ }^{1,2^{*}}$, FANGQIN YU ${ }^{1,2^{*}}$, RUNSHENG MA ${ }^{1,2}$, LELE ZHANG ${ }^{1,2}$, \\ GONGBO DU ${ }^{1,2}$, DONGPENG NIU ${ }^{1,2}$ and DETAO YIN ${ }^{1,2}$ \\ ${ }^{1}$ Department of Thyroid Surgery, The First Affiliated Hospital of Zhengzhou University; \\ ${ }^{2}$ Key Discipline Laboratory of Clinical Medicine of Henan, Zhengzhou, Henan 450052, P.R. China
}

Received November 8, 2020; Accepted June 30, 2021

DOI: $10.3892 / o r .2021 .8150$

\begin{abstract}
The present study was designed to observe the expression of the centrosomal protein 63 in papillary thyroid cancer (PTC) tissues and cells and to explore the clinical significance of Cep63 expression in PTC. Primary PTC tissues and matched normal thyroid tissues were collected, and the Cep63 expression level was determined by reverse transcription-quantitative PCR and western blotting. A stable Cep63-knockout cell line was constructed to assess the proliferation, invasion, migration and apoptosis abilities in vitro. A subcutaneous tumorigenesis model was established in nude mice to evaluate the effect of Cep63 on tumor growth and proliferation in vivo. Western blotting was used to explore the relevant signaling pathways. The results revealed that the expression level of Cep63 in PTC tissues was significantly increased. The proliferation, invasion and migration abilities of TPC-1 cells were decreased after Cep63 knockout, and silencing of Cep63 resulted in TPC-1 cell cycle arrest in the S phase. Mechanistically, Cep63 knockout inhibited the activation of the Janus kinase/signal transducer and activator of transcription 3 signaling pathway. In conclusion, Cep63 knockout significantly inhibited biological functions of TPC-1 cells in vitro and in vivo, indicating that Cep63 may be an important oncogene of PTC.
\end{abstract}

\section{Introduction}

Thyroid cancer accounts for approximately $1 \%$ of all human malignancies and $90 \%$ of endocrine malignancies (1).

Correspondence to: Professor Detao Yin, Department of Thyroid Surgery, The First Affiliated Hospital of Zhengzhou University, 1 Jianshe East Road, Zhengzhou, Henan 450052, P.R. China E-mail: detaoyin@zzu.edu.cn

\section{${ }^{*}$ Contributed equally}

Key words: centrosomal protein 63, papillary thyroid cancer, proliferation, invasion, Janus kinase/signal transducer and activator of transcription 3
Papillary thyroid cancer (PTC) is the most common pathological type and accounts for approximately $70-80 \%$ of all thyroid cancers (2-5). Most PTCs can be treated with thyroid surgery alone or combined with radioactive iodine therapy. Although numerous genetic changes have been revealed in thyroid cancer, the molecular mechanism of PTC progression has not been fully studied. Therefore, further study of the mechanism by which PTC occurs and develops is urgently needed.

Centrosomes are the microtubule-organizing centers of animal cells that ensure normal behavior of chromosomes during mitosis, so their number must be precisely controlled (6). The mutant products of some protooncogenes and tumor suppressor genes can directly affect the functional and structural integrity of centrosomes $(7,8)$. Previous studies have revealed that centrosomal aberrancies promote tumorigenesis, indicating that centrosome amplification is closely related to the development of cancer $(9,10)$. In addition, centrosome amplification is suspected to represent an early event of tumorigenesis (11). Research has confirmed that centrosomal aberrancies are prevalent in breast $(12,13)$, colon (6), bladder (14), pancreatic (15), prostate (16) as well as other cancer cell lines.

Some studies have reported that the number of centrioles is strictly regulated by centrosomal protein 63 (Cep63), Cep152, Cep131 and polo-like kinase 4 (Plk4) through the ubiquitin (Ub)-proteasome protein degradation system $(6,17,18)$. As the precursor of centrosomes, Cep63 is located on chromosome $3 \mathrm{q} 22.2$ and encodes a $63-\mathrm{kDa}$ protein with six coiled-coil domains; it is known for its ability to bind Cep152 to form the annular structural base of the centrosome (19). A previous study (20) revealed that Cep63 is involved in spindle assembly and DNA damage after spindle inactivation, binding to centrosomes and regulating mitosis, and additionally causes chromosomal instability and aberrations that lead to poor prognosis and potentially cancer-related overexpression. Cep63 has been revealed to promote the development and evolution of tumors (21); however, the relationship between Cep63 and PTC development remains unclarified. In the present study, Cep63 was knocked out via CRISPR/Cas9 technology to clarify its influence on TPC-1 cell behavior. The effects of Cep63 on the Janus kinase/signal transducer and activator of transcription 3 (JAK/STAT3) signaling pathway in TPC-1 cells was further investigated. 


\section{Materials and methods}

Patient tissue samples. To study the clinicopathological function of Cep63 in PTC, a total of 140 patients were recruited from the Department of Thyroid Surgery of the First Affiliated Hospital of Zhengzhou University (Zhengzhou, China) from November 2019 to March 2020 and participated in the trial. Inclusion criteria were patients with pathologically confirmed PTC, and exclusion criteria were patients with confirmed PTC who had received other treatments prior to surgery. There were 39 males and 101 females. The mean age of these patients was $51.89 \pm 0.7850$ years $(n=140)$. All participating patients were informed about the study and signed informed consent forms. The present study was also approved (approval no. 2019-KY-313) by the Ethics Committee for Scientific Research and Clinical Trial of the First Affiliated Hospital of Zhengzhou University and conducted in accordance with The Declaration of Helsinki Principles (version 2013). A total of 140 tumor tissues and 140 paired normal thyroid tissues were collected and stored in a refrigerator at $-80^{\circ} \mathrm{C}$ immediately after being obtained from patients with confirmed PTC undergoing thyroid surgery at The First Affiliated Hospital of Zhengzhou University.

Cell line and cell culture. The human PTC cell line TPC-1 was kindly provided by Dr Ye Lei of Shanghai Ruijin Hospital (Shanghai, China). To provide an experimental basis for studying the functions of Cep63, the CRISPR/Cas9 technique (Shanghai GeneChem Co., Ltd.) was used to construct a stable Cep63 knockout TPC-1 cell line. Cas-Designer, a web-based tool for choice of CRISPR-Cas9 target sites (http://www.rgenome.net/cas-designer/), was used to design the CEP63-sgRNA sequence. CEP63-sgRNA is located in the third exon of the CEP63 gene. The sequence of the CEP63-sgRNA was as follows: AGCTTCAGAAAAAGC AAATG. The transfected Cas9 plasmid, CEP63-sgRNA, is a custom-made product commissioned by Shanghai GeneChem Co., Ltd. There were two mutant genotypes in CEP63 allele with deletion of DNA level at the target action site. The corresponding deletion sequences were AATTCCCTCATT TG and AT. Cells were maintained in RPMI-1640 culture medium (Beijing Solarbio Science \& Technology Co., Ltd.) supplemented with $10 \%$ fetal bovine serum (FBS; Gemini Bio-Products, Inc.) and incubated at $37^{\circ} \mathrm{C}$ in a humidified atmosphere containing $5 \% \mathrm{CO}_{2}$. In the experiments with inhibitor interference, cells in the experimental group were cultured at $37^{\circ} \mathrm{C}$ in complete medium containing $10 \mathrm{nmol} / 1 \mathrm{LY} 2784544$ (GlpBio Technology, Inc.), while the culture medium of cells in the control group contained an equal concentration of dimethyl sulfoxide (DMSO).

Colony formation assay. TPC-1 cells were digested and plated in the culture dish at a concentration of 400 cells/well. The cells were divided into the TPC-1 group and the Cep63-KO group, with 3 replicates in each group. The cells were thoroughly mixed and incubated at $37^{\circ} \mathrm{C}$, and the medium was replaced with fresh medium every 2 days. When the cultured cells had formed visible cell colonies ( $>50$ cells/colony), the cells were fixed with $4 \%$ paraformaldehyde for $20 \mathrm{~min}$ at room temperature, and stained with $0.2 \%$ crystal violet for $30 \mathrm{~min}$ at room temperature. Cell counting was performed using a light microscope at a magnification of $\mathrm{x} 4$ and IBM SPSS 21.0 was used for statistical analysis.

Cell Counting Kit-8 (CCK-8) assay. Suspensions of digested cells in the logarithmic growth phase were uniformly inoculated into 96-well plates at a density of $1 \times 10^{4}$ cells $/ \mathrm{ml}$, and $100 \mu 1$ of the suspension was added to each well to establish 3 replicate wells. After the cells were completely attached to the bottom of the wells, $10 \mu \mathrm{l}$ of CCK- 8 detection reagent (Dojindo Molecular Technologies, Inc.) was added to each well. The culture plate was placed at $37^{\circ} \mathrm{C}$ and incubated for $2 \mathrm{~h}$. Then, the culture plate was placed in a Universal Microplate Reader and the absorbance was measured at $450 \mathrm{~nm}$.

Cell cycle analysis and apoptosis assay. The following protocol was followed for cell cycle analysis: Logarithmic growth phase cells were seeded in a culture flask. The cells were harvested by digestion with a trypsin solution containing $0.25 \%$ EDTA. The cell concentration was adjusted to $1 \times 10^{6}$ cells $/ \mathrm{ml}$. One $\mathrm{ml}$ of the cell suspension was centrifuged at $100 \mathrm{x} \mathrm{g}$ for $3 \mathrm{~min}$ at room temperature; the upper layer of medium was removed, and cell suspension was fixed in $500 \mu \mathrm{l}$ cold $70 \%$ ethanol at $4^{\circ} \mathrm{C}$ overnight. Then, the cells were washed with PBS to remove the fixation solution. The prepared $500 \mu \mathrm{l}$ PI/RNase A staining solution (Nanjing KeyGen Biotech Co., Ltd.) was added to the cells, which were incubated in the dark for $30 \mathrm{~min}$ at room temperature and then detected by flow cytometry (model: XL-MCL ADC; Beckman Coulter, Inc.). The following protocol was followed for the apoptosis assay: The cells were digested with trypsin without EDTA and counted at approximately $2 \times 10^{5}$ cells $/ \mathrm{ml}$. Next, subsequent staining was performed with the same volume of Annexin V-FITC and propidium iodide (PI) for $15 \mathrm{~min}$ at room temperature. The procedures were performed with an Annexin V-FITC Apoptosis Detection Kit (cat. no. KGA105-KGA108; Nanjing KeyGen Biotech Co., Ltd.) in accordance with the kit instructions. GraphPad Prism 6.0 (GraphPad Prism Software, Inc.) was used for analysis.

Transwell invasion assay. The Transwell assay was performed with 24-well culture plates and the matching Transwell upper chamber inserts ( $8 \mu \mathrm{m}$ pore size; Corning Inc.). Matrigel matrix (BD Biosciences) was prediluted in serum-free RPMI-1640 medium and evenly spread in the upper chamber at $37^{\circ} \mathrm{C}$ for $4 \mathrm{~h}$; the plates were then incubated in the incubator at $37^{\circ} \mathrm{C}$ for $6 \mathrm{~h}$. A total of $2 \times 10^{4}$ cells was mixed into $200 \mu \mathrm{l}$ of serum-free RPMI-1640 medium and inoculated into the upper chambers, and $600 \mu \mathrm{l}$ of RPMI-1640 medium supplemented with $10 \%$ FBS was added to the lower chambers. The Transwell inserts were fixed with $4 \%$ paraformaldehyde for $15 \mathrm{~min}$ and stained with $0.1 \%$ crystal violet for $30 \mathrm{~min}$ at room temperature. Finally, the migrated cells were counted using a fluorescence inverted microscope (Olympus Corporation) at a magnification of $x 200$.

Wound healing assay. Cells were uniformly inoculated in a 6 -well plate at a concentration of $5 \times 10^{5}$ cells/well. When the cells were cultured to $>95 \%$ confluence, sterile tips were used to create a scratch in each well. Next, the suspended cell 
fragments were removed by washing with sterile PBS, and the cells were cultured in serum-free RPMI-1640 medium at $37^{\circ} \mathrm{C}$ for $24 \mathrm{~h}$. Then, images of the healing of the cell scratches at the same position in each well were captured using a light inverted microscope (Olympus Corporation) at a magnification of x10. The area of the wound was assessed by ImageJ $1.42 \mathrm{q}$ software (National Institutes of Health), and the ratio of the area covered by the cells after migration to the area of the wound at the beginning of the experiment was calculated to compare the migration ability of the cells.

Reverse transcription-quantitative $(R T-q) P C R$ analysis. Total RNA was isolated from TPC-1 cells and nude mouse tissues with TRIzol reagent (Invitrogen; Thermo Fisher Scientific, Inc.) following the manufacturer's instructions. Total RNA was reversely transcribed to cDNA with a RevertAid First Strand cDNA Synthesis Kit (Takara Bio, Inc.) according to the manufacturer's protocol. Each reaction for quantitative analysis was performed according to the instructions for SYBR ${ }^{\circledR}$ Green Fast qPCR Mix (Wuhan Servicebio Technology Co., Ltd.). The PCR cycles were performed as follows: One cycle at $95^{\circ} \mathrm{C}$ for $10 \mathrm{~min}, 40$ cycles at $95^{\circ} \mathrm{C}$ for $15 \mathrm{sec}, 60^{\circ} \mathrm{C}$ for $60 \mathrm{sec}$, and one final cycle at $72^{\circ} \mathrm{C}$ for $10 \mathrm{~min}$, followed by cooling to $4^{\circ} \mathrm{C}$. $\beta$-actin was used as the reference standard for each cDNA sample. The primer sequences were as follows: $\beta$-actin forward, 5'-CACCCAGCACAATGAAGATCAAGA T-3' and reverse, 5'-CCAGTTTTTAAATCCTGAGTCAAG C-3'; and Cep63 forward, 5'-AGGAAGGCTCTGGCTGAA CAAT-3' and reverse, 5'-GTCATTAGCCCGCTCCAGTTT-3'. The mRNA expression levels were calculated as relative fold changes based on the $2^{-\Delta \Delta \mathrm{Cq}}$ method (22).

Western blotting. The TPC-1 cells were lysed in RIPA lysis buffer (Leagene, China). Protein concentration was determined by the BCA assay. Each lane was loaded with $10 \mu$ l protein. Protein from each sample was isolated by $10 \%$ SDS-PAGE (Leagene Biotech Co., Ltd.) and transferred to a PVDF membrane (EMD Millipore), followed by blocking in 5\% milk for $2 \mathrm{~h}$ at room temperature. The membrane was incubated with the primary antibodies Cep63 (1:2,000; product code ab235513; Abcam), JAK2 (1:5,000; product code ab108596; Abcam), p-JAK (1:2,000; product code ab32101; Abcam), STAT3 (1:1,000; product no. 9139T; Cell Signaling Technology, Inc.), p-STAT3 (1:2,000; product no. 9145T; Cell Signaling Technology, Inc.) and actin (1:1,000; cat. no. GB12001; Wuhan Servicebio Technology Co., Ltd.) overnight at $4{ }^{\circ} \mathrm{C}$ and washed 3 times with TBS containing $0.1 \%$ Tween-20 (Leagene Biotech Co., Ltd.) at room temperature. Then, the membrane was incubated with an HRP-conjugated IgG secondary antibody (cat. nos. SA00001-2 and SA00001-1; ProteinTech Group, Inc.) for $1 \mathrm{~h}$ at room temperature and visualized with an ECL detection kit (Thermo Fisher Scientific, Inc.). The protein expression levels were assessed by band densities using AlphaEaseFC ${ }^{\text {тм }} 4.0$ (ProteinSimple) software.

In vivo tumorigenicity assay in nude mice. A total of 20 female BALB/c nude mice (4 weeks old; 16-18 g) purchased from Beijing Vital River Laboratory Animal Technology Co., Ltd. were used to establish the animal model of subcutaneous neoplasia. Based on animal welfare considerations, the mice were housed in specific pathogen-free housing at $27^{\circ} \mathrm{C}$ with a humidity of 50\% and a 14/10-h light/night cycle. Food and water were continuously provided throughout the day at Henan Key Laboratory for Pharmacology of Liver Diseases. Each mouse was subcutaneously inoculated in the right flank in a total volume of $200 \mu \mathrm{l}$ resuspension solution at a density of $1 \times 10^{7}$ cells $/ \mathrm{ml}$. The mice were weighed and their health and behaviour were observed once a day. They were sacrificed by cervical dislocation after $\mathrm{CO}_{2}$ anesthesia (flow rate of $\mathrm{CO}_{2}$ : $16.67 \%$ volume displacement $/ \mathrm{min}$ ) when body weight loss $>2 \mathrm{~g}$ or the maximum diameter of the tumor was over $20 \mathrm{~mm}$. None of the mice had any other special conditions or other causes of death during the study. Finally, the tumor growth and inhibition rates were calculated. The animal trials lasted a total of two weeks. The present study was approved (approval no. 2019-KY-313) by the Ethics Committee for Scientific Research and Clinical Trial of the First Affiliated Hospital of Zhengzhou University (Zhengzhou, China).

Statistical analysis. GraphPad Prism 6.0 (GraphPad Prism software, Inc., USA) was used for analysis. Data comparisons between two groups were performed with unpaired Student's t-test, comparisons between tumor and adjacent non-tumor tissues were performed with paired t-test. Comparisons among multigroups were performed with one-way analysis of variance (ANOVA), and then Bonferroni test was applied as a post hoc test. The results are presented as the means \pm standard deviations (SDs). The association between the Cep63 expression level and potential pathological factors was assessed with the chi-square test. $\mathrm{P}<0.05$ was considered to indicate a statistically significant difference. The experiments were repeated in triplicate.

\section{Results}

Cep63 is highly expressed in PTC tissues. The results in Fig. 1A revealed that Cep63 expression was significantly higher in PTC tissues than in normal thyroid tissues. According to the eighth edition of the American Joint Committee on Cancer/Union for International Cancer Control tumor-node-metastasis staging system (TNM-8) $(23,24)$ and using the median expression level of Cep63 as the cutoff point, the patients were divided into the high-expression and low-expression groups and the relationship between the expression level of Cep63 and the clinicopathological features was further analyzed. A significant association was revealed between high Cep63 expression and lymph node metastasis of PTC $(\mathrm{P}=0.017)$ (Table I). However, the age, sex, tumor size, TNM stage and BRAF mutation status of the patients were not significantly associated with the Cep63 expression level $(\mathrm{P}>0.05)$. In general, $\mathrm{Cep} 63$ is highly expressed in PTC and is probably involved in the progression of PTC.

Knockout of Cep63 in TPC-1 cells. To explore the function of Cep63 in TPC-1 cells, CRISPR/Cas9 editing was used to construct Cep63-KO cell line. RT-qPCR and western blotting were used to assess the expression of Cep63 at the protein and mRNA levels, respectively, to verify the knockout efficiency in these cells. The results of these analyses revealed that Cep63 expression was abolished in the Cep63-KO cell line generated by CRISPR/Cas9 editing of TPC-1 cells (Fig. 1B and C). 
Table I. Association between Cep63 expression and clinicopathological features in papillary thyroid cancer.

\begin{tabular}{|c|c|c|c|c|}
\hline \multirow[b]{2}{*}{ Variables } & \multirow[b]{2}{*}{ No. of cases } & \multicolumn{2}{|c|}{ Cep63 expression } & \multirow[b]{2}{*}{ P-value } \\
\hline & & High & Low & \\
\hline Sex & & & & 0.346 \\
\hline Male & 39 & 17 & 22 & \\
\hline Female & 101 & 53 & 48 & \\
\hline Age (years) & & & & 0.73 \\
\hline$<55$ & 84 & 43 & 41 & \\
\hline$\geq 55$ & 56 & 27 & 29 & \\
\hline Tumor size $(\mathrm{cm})$ & & & & 0.17 \\
\hline$<2$ & 82 & 37 & 45 & \\
\hline$\geq 2$ & 58 & 33 & 25 & \\
\hline Lymph node metastasis & & & & 0.017 \\
\hline No & 62 & 24 & 38 & \\
\hline Yes & 78 & 46 & 32 & \\
\hline TNM stage & & & & 0.368 \\
\hline I & 97 & 46 & 51 & \\
\hline II & 34 & 19 & 15 & \\
\hline III-IV & 9 & 5 & 4 & \\
\hline BRAF mutation & & & & 0.338 \\
\hline No & 37 & 16 & 21 & \\
\hline Yes & 103 & 54 & 49 & \\
\hline
\end{tabular}

Cep, centrosomal protein.
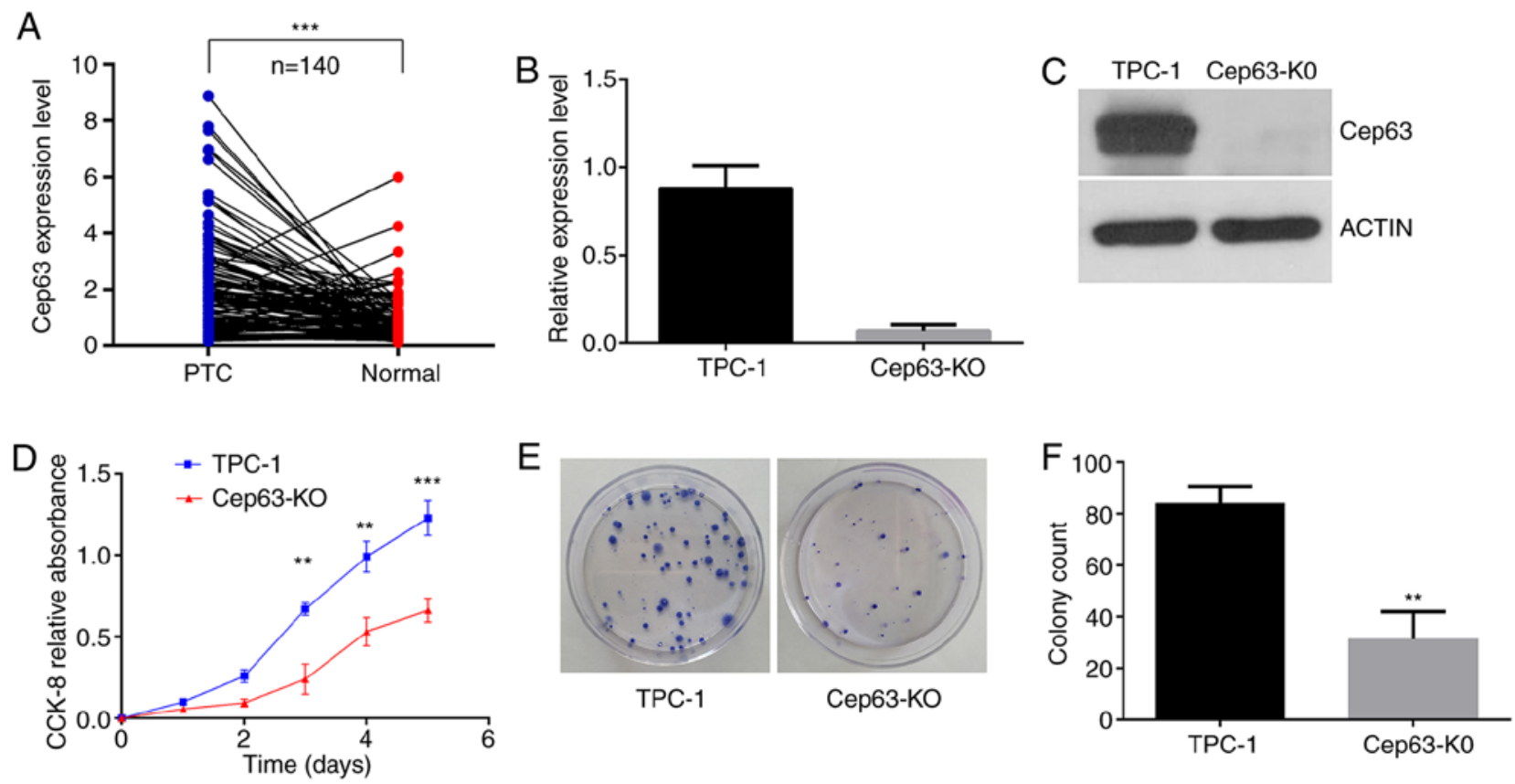

Figure 1. Cep63 is highly expressed in PTC tissues and Cep63 knockout inhibits proliferation of TPC-1 cells. (A) Expression of Cep63 in tissues was detected by RT-qPCR. (B and C) RT-qPCR and western blotting were used to confirm the deficient expression of Cep63 in TPC-1 cells edited with CRISPR/Cas9 technology. (D-F) Cell proliferation was assessed by Cell Counting Kit- 8 and colony formation assays. The data are expressed as the means \pm SDs. ${ }^{* *} \mathrm{P}<0.01$ and ${ }^{* * * *} \mathrm{P}<0.001$ compared with the control. PTC, papillary thyroid cancer; RT-qPCR, reverse transcription-quantitative PCR; KO, knockout.

Knockout of Cep63 inhibits the proliferation of TPC-1 cells. The CCK-8 method was used to assess the proliferation ability of the two groups of cells. Comparison between the two groups revealed that the growth trend of Cep63-KO cells was relatively 

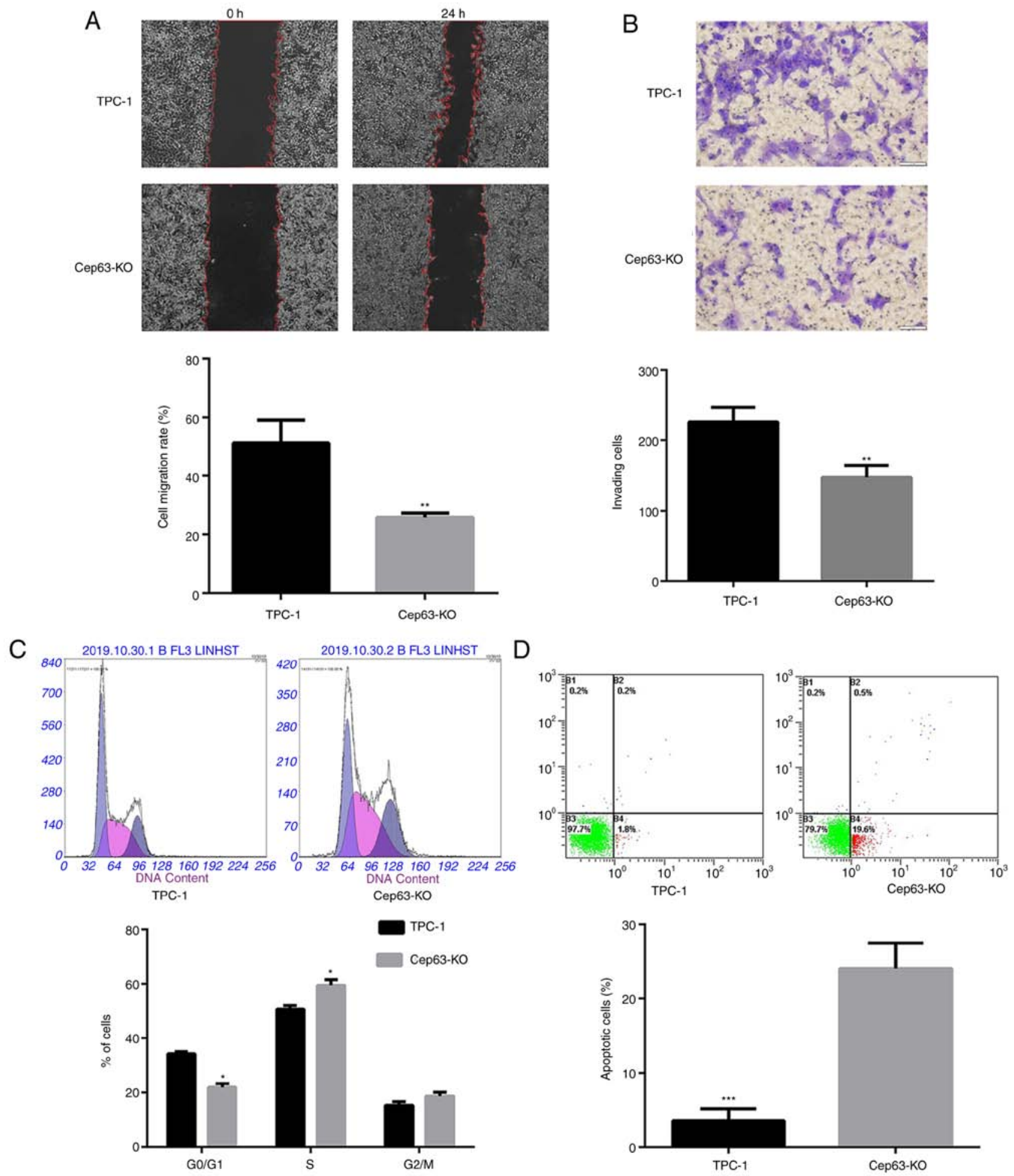

Figure 2. Cep63 knockout inhibits the malignant phenotypes of TPC-1 cells in vitro. (A) Migration was assessed by wound healing assay. (B) A Transwell assay was used to detect cell invasion. (C) The cell cycle distribution was assessed by flow cytometry. (D) The apoptotic rate was assessed by flow cytometry. The data are expressed as the means \pm SDs. ${ }^{*} \mathrm{P}<0.05,{ }^{* * *} \mathrm{P}<0.01$ and ${ }^{* * * *} \mathrm{P}<0.001$ compared with the control. KO, knockout.

mild and that the growth rate of these cells was significantly lower than that of TPC-1 cells $(\mathrm{P}<0.001$; Fig. 1D), indicating that Cep63 knockout inhibited the proliferation of TPC-1 cells. In addition, TPC-1 cell colony formation rate of $21 \pm 0.95 \%$ and the Cep63-KO cell colony formation rate of $7.83 \pm 1.53 \%$ were significantly different $(\mathrm{P}<0.01$; Fig. $1 \mathrm{E}$ and $\mathrm{F})$, indicating that Cep63 knockout obviously reduced the rate of colony formation of TPC-1 cells.
Knockout of Cep63 suppresses the migration and invasion of TPC-1 cells. The effect of Cep63 knockout on the functional characteristics of TPC-1 cells was evaluated by cell migration and invasion experiments. Cep63 knockout reduced the cell migration rate by $25.31 \%$ compared with that of TPC-1 cells $(\mathrm{P}<0.01$; Fig. $2 \mathrm{~A})$. In addition, after Cep63 knockout, the number of cells penetrating the Transwell membrane was significantly lower than that of TPC-1 cells $(147.0 \pm 10.12$ and 


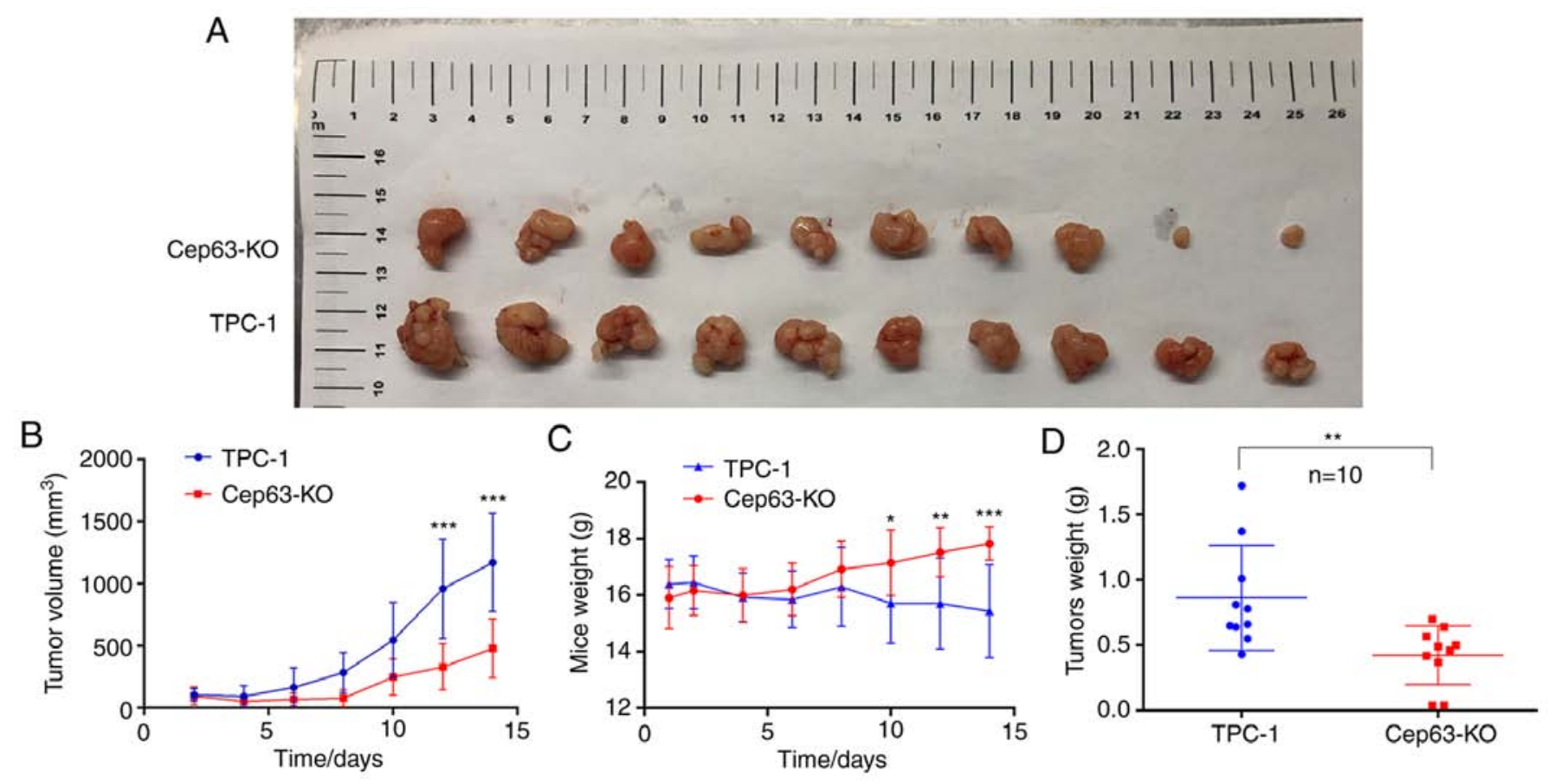

Figure 3. Cep63 knockout inhibits proliferation of TPC-1 cells in vivo. (A) Images of tumors from each group. (B) Volume of tumors. (C) Weight of mice. (D) Weight of tumors. The data are expressed as the means \pm SDs. ${ }^{*} \mathrm{P}<0.05,{ }^{* *} \mathrm{P}<0.01$ and ${ }^{* * * *} \mathrm{P}<0.001$ compared with the control. KO, knockout; d, day.

226.3 $\pm 11.89 ; \mathrm{P}<0.01$ ) (Fig. 2B). These data indicated that Cep63 mediated the malignant invasion and migration of TPC-1 cells.

Knockout of Cep63 induces $S$-phase cell cycle arrest and promotes the apoptosis of TPC-1 cells. The flow cytometric results revealed that compared with TPC-1 cells, Cep63-KO cells exhibited a significantly decreased population in the $\mathrm{G}_{0} / \mathrm{G}_{1}$ phase and a significantly increased population in the $\mathrm{S}$ phase (Fig. 2C). These results indicated that the transition of TPC-1 cells from the $G_{0} / G_{1}$ phase to the $S$ phase was significantly $(\mathrm{P}<0.05)$ increased after Cep63 knockout, suggesting that Cep63 may play an important role in regulating the cell cycle in TPC-1 cells. Furthermore, the apoptotic rate of Cep63-KO cells was significantly increased compared with that of TPC-1 cells (20.1 and 2\%, respectively; Fig. 2D), indicating the important role of Cep63 in mediating the apoptosis of TPC-1 cells.

Cep63 stimulates tumor proliferation activity in vivo. The effect of Cep63 on the growth of TPC-1 cells was confirmed in vivo by a subcutaneous tumor formation assay in nude mice. The tumors in the Cep63-KO group had smaller volumes and grew more slowly than those in the TPC-1 group $(\mathrm{P}<0.001$; Fig. 3A and B). The weight of tumors followed a similar pattern: The tumors in the Cep63-KO group weighed significantly less than those in the TPC-1 group $(\mathrm{P}<0.01$; Fig. 3D), indicating that Cep63 also actively regulated the growth of TPC-1 cells in vivo. Furthermore, these results vividly revealed that as the tumors grew, the weight of the mice in the TPC-1 group decreased significantly, while that of the mice in the Cep63-KO group increased gradually ( $\mathrm{P}<0.001$; Fig. 3C). It was hypothesized that this pattern may have resulted from cachexia caused by the malignant proliferation behavior of
TPC-1 cells in mice, which also indicated that Cep63 mediated the malignant proliferation of TPC-1 cells.

JAK/STAT3 signaling pathway may be involved in the Cep63-mediated malignant behavior of TPC-1 cells. The expression of the JAK/STAT3 pathway was studied through western blotting to explore the molecular mechanism of Cep63 in TPC-1 cells. The p-JAK and p-STAT3 levels were decreased by 11.6 and $33.0 \%$, respectively, in Cep63-KO group compared with TPC-1 group (Fig. 4). After treatment with the JAK inhibitor LY2784544 for $24 \mathrm{~h}$ in TPC-1+LY2784544 group, as expected, the protein levels of p-JAK and p-STAT3 were significantly decreased by 25.2 and $27.6 \%$ compared with the TPC-1 cell group, respectively. The results indicated that JAK/STAT3 may be involved in the Cep63-mediated malignant behavior of TPC-1 cells.

\section{Discussion}

Centrosomal proteins play an important role in molecular recognition in the regulation of cell function and activity. These proteins may be important in human tumorigenesis and provide new targets for the diagnosis and treatment of tumors $(25,26)$. A previous study (19) demonstrated that Cep63 promotes the replication of centrioles and that Cep63 knockout causes centrosomal distortion, chromosomal entanglement and telomere clustering defects, indicating that centrosomes mediate the regulation of the DNA damage response.

The Cep63 expression was strongly upregulated in PTC tissues and lymph node metastasis was associated with high Cep63 expression; these results were consistent with an oncogenic role for the Cep63 gene in PTC. BCPAP is a mutant cell line, which was initially selected, however, since other types of cell lines, such as BCPAP, could not be obtained through 

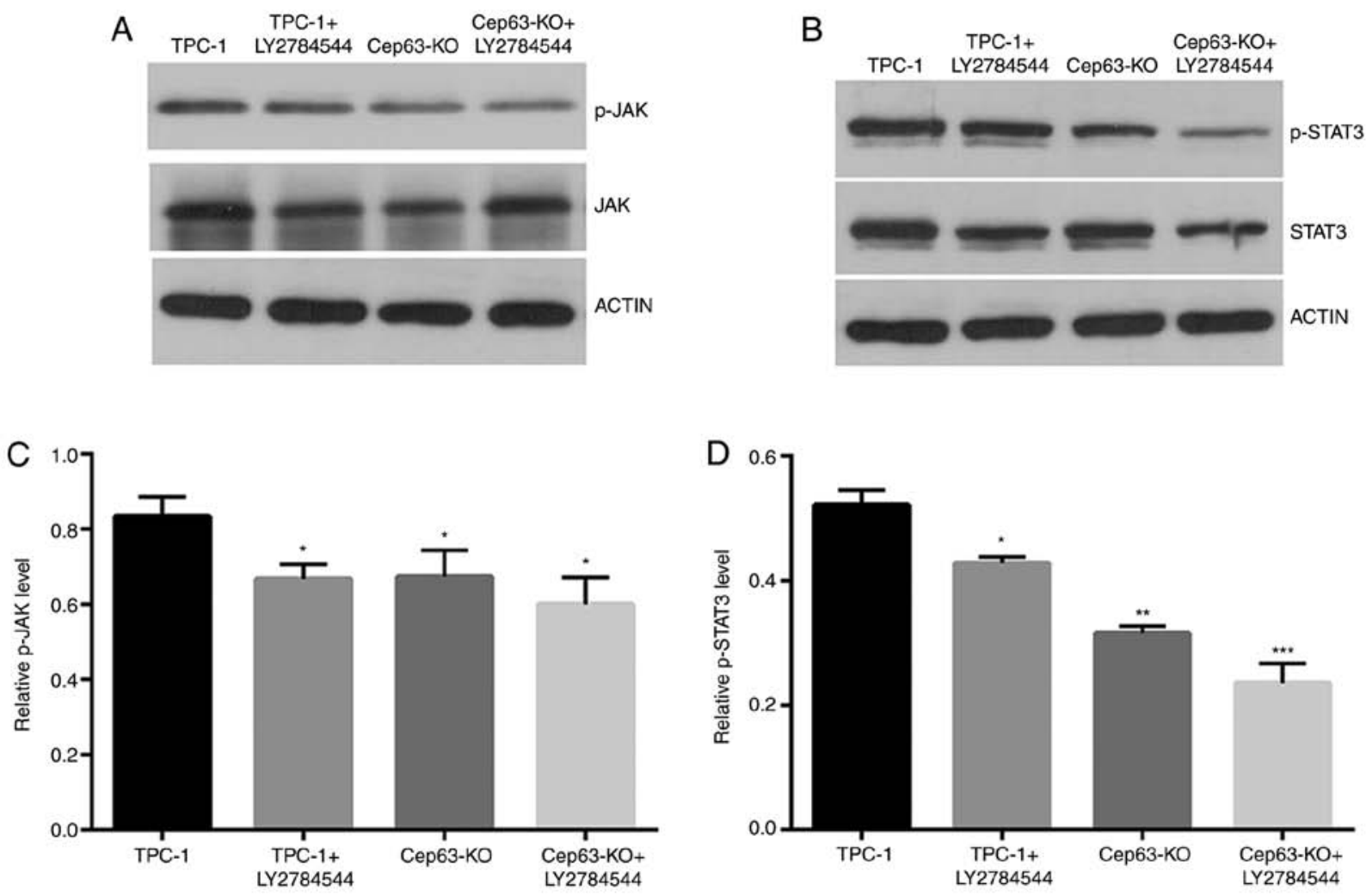

Figure 4. Cep63 knockout inhibits the JAK/STAT3 pathway. (A and B) Cells were treated or not with JAK/STAT inhibitor LY2784544 and the protein level was assessed by western blotting. (A and C) The expression of p-JAK. (B and D) The expression of p-STAT3. The data are expressed as the means \pm SDs. ${ }^{*} \mathrm{P}<0.05,{ }^{* *} \mathrm{P}<0.01$ and ${ }^{* * * *} \mathrm{P}<0.001$ compared with the control. JAK/STAT3, Janus kinase/signal transducer and activator of transcription 3; $\mathrm{p}-$, phosphorylated; KO, knockout.

effective ways, and considering that the TPC-1 cell line has been widely used in previous research (27), the TPC-1 cell line was finally selected and focus was addressed on the effect of the CEP63 gene on the TPC-1 cell line. The biological function of Cep63 was further studied in subsequent experiments. It was revealed that the proliferation, migration and invasion of Cep63-KO cells was significantly suppressed compared with that of TPC-1 cells, and that Cep63 knockout promoted cell cycle progression from the $\mathrm{G}_{0} / \mathrm{G}_{1}$ phase to the $\mathrm{S}$ phase. The data also revealed that Cep63 knockout promoted TPC-1 cell apoptosis. In vivo experiments further confirmed that Cep63 knockout decreased the growth rate and size of tumors compared with those in the TPC-1 group and even resulted in a significant difference in the weight of mice between two groups. The present study revealed that Cep63 promoted TPC-1 cell proliferation, migration and invasion and inhibited apoptosis both in vivo and in vitro, consistent with results identifying Cep63 as an oncogene (21) and indicating the key role of Cep63 in PTC. However, due to COVID-19 and other unavoidable reasons, it has not been possible to collect xenograft tumor tissues in time to continue the detection of tumor markers.

The JAK/STAT3 signaling pathway is a widely recognized carcinogenic pathway and plays a key role in mediating proliferation, invasion and migration in some cancers $(28,29)$. STAT3 has been revealed to be targeted by the signaling of numerous receptor and nonreceptor tyrosine kinases in cancer cells. In addition, it is a transcription factor that regulates the expression of a wide range of genes and recruits tumor progression-promoting immune cells into the tumor microenvironment (30). After Cep63 knockout or JAK inhibitor treatment in TPC-1 cells, the expression levels of both p-JAK and p-STAT3 were decreased, consistent with earlier reports (31-33) that STAT3 is upregulated in human PTC tissues and that the JAK/STAT3 pathway plays a role in promoting the proliferation and migration of cancers. These data indicated that the JAK/STAT3 signaling pathway may be involved in the Cep63-mediated malignant biological behavior of TPC-1 cells. In a previous study (34), a degree of difference was detected in the expression of overall JAK and STAT3 as well as phosphorylated JAK and STAT3, and the difference was statistically significant. During the experiment, every effort was made to keep other controllable factors consistent, and it was initially hypothesized that the expression difference of Cep63 may affect the expression of JAK and STAT3 proteins. However, the specific molecular mechanism still needs to be further studied.

In conclusion, the results of the present study revealed that blocking the expression of Cep63 inhibited the proliferation, migration and invasion and promoted apoptosis of TPC-1 cells and that the JAK/STAT pathway may be strongly involved. The preliminary discussion of the role of Cep63 in promoting the malignant behavior of TPC-1 cells was provided, and it was suggested that Cep63 may be a potential oncogene for PTC cell line TPC-1. There have been no studies on the relationship between Cep63 and thyroid cancer progression, to the best of our knowledge. The BCPAP cell line is going to be obtained in order to study whether the 
tumor-promoting role of Cep63 is BRAF-dependent or not. Since other pathological types of thyroid cancers are relatively rare clinically, a certain number of clinical specimens cannot be collected in the short term at present. In future, other types of thyroid cancers will be gradually collected, and then the relationship between other types of thyroid cancers and the expression of Cep63 will be examined.

\section{Acknowledgements}

The authors would like to thank Professor Qingduan Wang (Henan Key Laboratory for Pharmacology of Liver Diseases) for proofreading the article and Dr Youmei Peng (Henan Key Laboratory for Pharmacology of Liver Diseases) for the guidance in the animal experiments.

\section{Funding}

The present study was supported by Technological Innovation Team Project of Henan Province (grant no. 19IRTSTHN002), The Thousand Talents Science and Technology Innovation Leading Talents Subsidy Project of Central Plains (grant no. 194200510011), the Major Scientific Research Projects of Traditional Chinese Medicine in Henan Province (grant no. 20-21ZYZD14) and the Cultivation of Young and Middle-aged Health Science and Technology Innovation Leading Talents in Henan Province (grant no. YXKC2020015).

\section{Availability of data and materials}

The datasets generated during and/or analyzed during the current study are available from the corresponding author on reasonable request.

\section{Authors' contributions}

CL and FY prepared the manuscript and designed the study. RM, LZ, GD and DN performed the experiments, analyzed the data and performed the literature research. DY was responsible for the process design, manuscript review for important intellectual content and integrity assurance of the whole study. All authors read and approved the final manuscript.

\section{Ethics approval and consent to participate}

All the experiments in the present study were approved (approval no. 2019-KY-313) by the Ethics Committee for Scientific Research and Clinical Trial of the First Affiliated Hospital of Zhengzhou University (Zhengzhou, China). Written informed consent was obtained from all patients prior to enrollment in the present study.

\section{Patient consent for publication}

Not applicable.

\section{Competing interests}

The authors declare that they have no competing interests.

\section{References}

1. Wang S, Wu J, Ren J, Vlantis AC, Li MY, Liu SYW, Ng EKW, Chan ABW, Luo DC, Liu Z, et al: MicroRNA-125b interacts with Foxp3 to induce autophagy in thyroid cancer. Mol Ther 26: 2295-2303, 2018.

2. Yin D, Wu W, Li M, Wang QE, Li H, Wang Y, Tang Y and Xing M: DKK3 is a potential tumor suppressor gene in papillary thyroid carcinoma. Endocr Relat Cancer 20: 507-514, 2013.

3. Dalal V, Kaur M and Bansal A: Papillary carcinoma thyroid with anastomosing channels: An unusual morphology. J Lab Physicians 9: 140-142, 2017.

4. Siegel RL, Miller KD and Jemal A: Cancer statistics, 2017. CA Cancer J Clin 67: 7-30, 2017.

5. Schneider DF and Chen H: New developments in the diagnosis and treatment of thyroid cancer. CA Cancer J Clin 63: 374-394, 2013.

6. Kim DH, Ahn JS, Han HJ, Kim HM, Hwang J, Lee KH, Cha-Molstad H, Ryoo IJ, Jang JH, Ko SK, et al: Cep131 overexpression promotes centrosome amplification and colon cancer progression by regulating Plk4 stability. Cell Death Dis 10: 570, 2019.

7. Fukasawa K: Oncogenes and tumour suppressors take on centrosomes. Nat Rev Cancer 7: 911-924, 2007.

8. Brinkley BR: Managing the centrosome numbers game: From chaos to stability in cancer cell division. Trends Cell Biol 11: 18-21, 2001.

9. Levine MS, Bakker B, Boeckx B, Moyett J, Lu J, Vitre B, Spierings DC, Lansdorp PM, Cleveland DW, Lambrechts D, et al: Centrosome amplification is sufficient to promote spontaneous tumorigenesis in mammals. Dev Cell 40: 313-322.e5, 2017.

10. Coelho PA, Bury L, Shahbazi MN, Liakath-Ali K, Tate PH, Wormald S, Hindley CJ, Huch M, Archer J, Skarnes WC, et al: Over-expression of Plk4 induces centrosome amplification, loss of primary cilia and associated tissue hyperplasia in the mouse. Open Biol 5: 150209, 2015.

11. Maric I, Viaggi S, Caria P, Frau DV, Degan P and Vanni R: Centrosomal and mitotic abnormalities in cell lines derived from papillary thyroid cancer harboring specific gene alterations. Mol Cytogenet 4: 26, 2011.

12. Pannu V, Mittal K, Cantuaria G, Reid MD, Li X, Donthamsetty S, McBride M, Klimov S, Osan R, Gupta MV, et al: Rampant centrosome amplification underlies more aggressive disease course of triple negative breast cancers. Oncotarget 6: 10487-10497, 2015.

13. Denu RA, Zasadil LM, Kanugh C, Laffin J, Weaver BA and Burkard ME: Centrosome amplification induces high grade features and is prognostic of worse outcomes in breast cancer. BMC Cancer 16: 47, 2016.

14. Kawamura K, Izumi H, Ma Z, Ikeda R, Moriyama M, Tanaka T, Nojima T, Levin LS, Fujikawa-Yamamoto K, Suzuki K and Fukasawa K: Induction of centrosome amplification and chromosome instability in human bladder cancer cells by p53 mutation and cyclin E overexpression. Cancer Res 64: 4800-4809, 2004.

15. Xie S, Qin J, Liu S, Zhang Y, Wang J, Shi X, Li D, Zhou J and Liu M: Cep70 overexpression stimulates pancreatic cancer by inducing centrosome abnormality and microtubule disorganization. Sci Rep 6: 21263, 2016.

16. Li J, Xuan JW, Khatamianfar V, Valiyeva F, Moussa M, Sadek A, Yang BB, Dong BJ, Huang YR and Gao WQ: SKA1 over-expression promotes centriole over-duplication, centrosome amplification and prostate tumourigenesis. J Pathol 234: 178-189, 2014.

17. Nigg EA, Cajanek L and Arquint C: The centrosome duplication cycle in health and disease. FEBS Lett 588: 2366-2372, 2014.

18. Watanabe Y, Honda S, Konishi A, Arakawa S, Murohashi M, Yamaguchi H, Torii S, Tanabe M, Tanaka S, Warabi E and Shimizu S: Autophagy controls centrosome number by degrading Cep63. Nat Commun 7: 13508, 2016.

19. Buim ME, Soares FA, Sarkis AS and Nagai MA: The transcripts of SFRP1,CEP63 and EIF4G2 genes are frequently downregulated in transitional cell carcinomas of the bladder. Oncology 69: 445-454, 2005

20. Smith E, Dejsuphong D, Balestrini A, Hampel M, Lenz C, Takeda S, Vindigni A and Costanzo V: An ATM- and ATR-dependent checkpoint inactivates spindle assembly by targeting CEP63. Nat Cell Biol 11: 278-285, 2009.

21. Loffler H, Fechter A, Matuszewska M, Saffrich R, Mistrik M, Marhold J, Hornung C, Westermann F, Bartek J and Krämer A: Cep63 recruits Cdk1 to the centrosome: Implications for regulation of mitotic entry, centrosome amplification, and genome maintenance. Cancer Res 71: 2129-2139, 2011. 
22. Livak KJ and Schmittgen TD: Analysis of relative gene expression data using real-time quantitative PCR and the 2(-Delta Delta C(T)) method. Methods 25: 402-408, 2001.

23. Zhi J, Wu Y, Hu L, Zhao J, Liu H, Ruan X, Hou X, Zhang J, Zheng $\mathrm{X}$ and Gao M: Assessment of the prognostic value and N1b changes of the eighth TNM/AJCC staging system for differentiated thyroid carcinoma. Int J Clin Oncol 25: 59-66, 2020.

24. Kim TH, Kim YN, Kim HI, Park SY, Choe JH, Kim JH, Kim JS Oh YL, Hahn SY, Shin JH, et al: Prognostic value of the eighth edition AJCC TNM classification for differentiated thyroid carcinoma. Oral Oncol 71: 81-86, 2017.

25. Li S, Hu X, Cui S and He D: Novel centrosome protein, TCC52, is a cancer-testis antigen. Cancer Sci 99: 2274-2279, 2008.

26. Kumar A, Rajendran V, Sethumadhavan R and Purohit R: CEP proteins: The knights of centrosome dynasty. Protoplasma 250: 965-983, 2013

27. Yin DT, Xu J, Lei M, Li H, Wang Y, Liu Z, Zhou Y and Xing M: Characterization of the novel tumor-suppressor gene CCDC67 in papillary thyroid carcinoma. Oncotarget 7: 5830-5841, 2016.

28. Liang C, Zhao T, Li H, He F, Zhao X, Zhang Y, Chu X, Hua C, Qu Y, Duan Y, et al: Long Non-coding RNA ITIH4-AS1 accelerates the proliferation and metastasis of colorectal cancer by activating JAK/STAT3 signaling. Mol Ther Nucleic Acids 18: 183-193, 2019.

29. Khanna P, Lee JS, Sereemaspun A, Lee H and Baeg GH: GRAMD1B regulates cell migration in breast cancer cells through JAK/STAT and Akt signaling. Sci Rep 8: 9511, 2018.
30. Yu H, Lee H, Herrmann A, Buettner R and Jove R: Revisiting STAT3 signalling in cancer: New and unexpected biological functions. Nat Rev Cancer 14: 736-746, 2014.

31. Notarangelo T, Sisinni L, Trino S, Calice G, Simeon V and Landriscina M: IL6/STAT3 axis mediates resistance to BRAF inhibitors in thyroid carcinoma cells. Cancer Lett 433: 147-155, 2018.

32. Chang Q, Bournazou E, Sansone P, Berishaj M, Gao SP, Daly L, Wels J, Theilen T, Granitto S, Zhang X, et al: The IL-6/JAK/Stat3 feed-forward loop drives tumorigenesis and metastasis. Neoplasia 15: 848-862, 2013.

33. Bi CL, Zhang YQ, Li B, Guo M and Fu YL: MicroRNA-520a-3p suppresses epithelial-mesenchymal transition, invasion, and migration of papillary thyroid carcinoma cells via the JAK1-mediated JAK/STAT signaling pathway. J Cell Physiol 234: 4054-4067, 2019.

34. Lu J, Zhang L, Zhou H, Du Z and Zhang G: Silencing of Girdin suppresses the malignant behavior of colorectal carcinoma cells. Oncol Rep 40: 887-894, 2018.

This work is licensed under a Creative Commons Attribution-NonCommercial-NoDerivatives 4.0 International (CC BY-NC-ND 4.0) License. 